\title{
苯并[a]萠对不同修复潜力羊茅属植物的根系分泌 物中几种低分子量有机物的影响
}

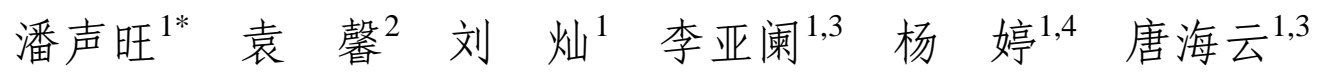

${ }^{1}$ 成都大学城乡建设学院, 成都 610106 ; ${ }^{2}$ 解放军后勤工程学院国防建筑与环境工程系, 重庆 401131; ${ }^{3}$ 云南农业大学资源环境学院, 昆明 $650201 ;{ }^{4}$ 西 南大学资源环境学院, 重庆 400715

摘 要 根系分泌物是植物与土壤间进行物质交换和信息传递的重要载体, 是植物响应外界胁迫的重要途径, 也是构成根际 微生态特征的关键因素。根系分泌物与有机污染物的植物修复密切相关, 研究胁迫条件下不同修复潜力植物间根系分泌物的 释放特征有助于揭示植物修复的内在机制。该文借助根际袋土培试验研究了苯并 $[\alpha]$ 萠(BaP)胁迫下 5 种羊茅属(Festuca)植物根 系不同生长期(30-70天)几种低分子量有机物的分泌特征。结果表明: 1) BaP浓度在10.25-161.74 mg $\mathrm{kg}^{-1}$ 范围内时, 待试植物 能有效地促进土壤中 $\mathrm{BaP}$ 的去除, 其修复潜力依次为苇状羊茅 $(F$. arundinacea $)>$ 草原羊茅 (F. chelungkiangnica $) \geq$ 毛稃羊 茅 (F. rubra subsp. arctica $) \geq$ 贫芒羊茅 $(F$. sinomutica $)>$ 细芒羊茅(F. stapfii)。2) BaP胁迫增强了植物根系对可溶性糖的分泌: 随着胁迫强度的增大、胁迫期的延长, 其分泌量变化呈“先升后降”趋势。3) BaP胁迫促进了植物根系低分子量有机酸的释放, 植物的修复潜力越大, 有机酸高峰值出现时的胁迫浓度越高; 组成成分较稳定, 草酸、乙酸、乳酸和苹果酸为主要组分 ( $>97.34 \%)$, 在修复潜力较强植物的根系分泌物中检测出微量的反丁烯二酸。4) BaP胁迫对氨基酸种类影响不大, 但对分泌量 影响较大。其中, 苏氨酸、丝氨酸、甘氨酸、丙氨酸的分泌量随 $\mathrm{BaP}$ 胁迫强度的增强而剧增; 脯氨酸、羟脯氨酸和天冬氨酸 近乎以加和效应甚至协同效应的形式参与植物对 BaP胁迫的应激反应: 参与应激组分的分泌量随胁迫强度的增强而剧增, 植 物的修复潜力越强, 参与的组分越多。可见BaP胁迫下, 5 种羊茅属植物根系分泌物中几种低分子量有机物的释放特征与植物 自身的修复潜力有关: 修复潜力越强, 释放量越多且成分也越复杂, 并呈现出较强的环境适应性及生理可塑性。

关键词 苯并 $[\alpha]$ 萠; 羊茅属植物; 根系分泌物; 低分子量有机物; 种间差异

引用格式: 潘声旺, 袁馨, 刘灿, 李亚阑, 杨婷, 唐海云 (2016). 苯并 $[\alpha]$ 萠对不同修复潜力羊茅属植物的根系分泌物中几种低分子量有机物的影响. 植物生态学报, 40, 604-614. doi: 10.17521/cjpe.2015.0426

\section{Effects of benzo [ $\alpha$ ] pyrene on the organic compounds of low molecule weight excreted by root systems in five Festuca species with different remediation potentials}

PAN Sheng-Wang ${ }^{1 *}$, YUAN Xin ${ }^{2}$, LIU Can ${ }^{1}$, LI Yan-Lan ${ }^{1,3}$, YANG Ting ${ }^{1,4}$ and TANG Hai-Yuan ${ }^{1,3}$

${ }^{1}$ Faculty of Urban and Rural Construction, Chengdu University, Chengdu 610106, China; ${ }^{2}$ Department of National Defense Architecture Planning \& Environmental Engineering, Logistical Engineering University of People's Liberation Army of China, Chongqing 401131, China; ${ }^{3}$ College of Resources and Environment, Yunnan Agricultural University, Kunming 650201, China; and ${ }^{4}$ College of Resources and Environment, Southwest University, Chongqing 400715, China

\section{Abstract}

Aims Root exudates have specialized roles in nutrient cycling and signal transduction between a root system and soil, as well as in plant responses to environmental stresses. They are the key regulators in the rhizosphere communications and can modify the biological and physical interactions between roots and soil organisms. Phytoremediation is an important measure to remove organic pollutants from contaminated soil, and root exudates are considered to be closely related to the mechanisms in the phytoremediation of soils contaminated by organic pollutants. This study was designed to determine the characteristics of root exudates in five Festuca species under the stress of benzo $[\alpha]$ pyrene (BaP) and to identify the effects of $\mathrm{BaP}$ on the organic compounds of low molecule weight in root exudates.

Methods Five Festuca species, which had been tested to be tolerant to the BaP stress, were used in this study. A soil-cultivating test, with rhizobag technique, was conducted to investigate the effect of BaP concentration on the organic compounds of low molecule weight in root exudates at different growth stages (30-70 days). The BaP

收稿日期Received: 2015-11-24 接受日期Accepted: 2016-04-23

*E-mail: panwang@swu.edu.cn 
concentrations in the contaminated soils were set for $10.25 \mathrm{mg} \cdot \mathrm{kg}^{-1}, 20.37 \mathrm{mg} \cdot \mathrm{kg}^{-1}, 40.45 \mathrm{mg} \cdot \mathrm{kg}^{-1}, 80.24$ $\mathrm{mg} \cdot \mathrm{kg}^{-1}$, and $161.74 \mathrm{mg} \cdot \mathrm{kg}^{-1}$ (denoted by $\mathrm{T}_{1}, \mathrm{~T}_{2}, \mathrm{~T}_{3}, \mathrm{~T}_{4}$ and $\mathrm{T}_{5}$, respectively).

Important findings The presence of vegetation enhanced the dissipation of $\mathrm{BaP}$ in soils. This effect was especially marked in treatment with $F$. arundinacea, followed sequentially by that of $F$. chelungkiangnica, $F$. rubra subsp. arctica and F. sinomutica; the dissipation of $\mathrm{BaP}$ in treatment with $F$. stapfi was lowest during the entire experiment. The contents of soluble sugars, organic acids, and amino acids in root exudates were all increased by the $\mathrm{BaP}$ treatments. The contents of soluble sugars in root exudates increased notably at relatively low BaP levels $\left(\mathrm{T}_{1}-\mathrm{T}_{3}\right)$ or in earlier stress stages (30-40 days), and declined at relatively high BaP levels $\left(\mathrm{T}_{4}-\mathrm{T}_{5}\right)$ or in later stress stages (40-70 days), with highest values always occurring in the $T_{3}$ treatments on day 50 of the experiments. In the five Festuca species, oxlic acid, acetic acid, lactic acid and malic acid are the main constituents of organic acids in root exudates, at greater than $97.34 \%$ in total in all treatments. However, there are traces of fumaric acid in the root exudates of Festuca species with stronger remediation potentials. When the contents of organic acids in root exudates reached the peak, the stronger the remediation potentials of plants were, the higher the concentrations of $\mathrm{BaP}$ would be to induce stress. Nineteen types of common amino acids were found in root exudates of Festuca and the proportion of total amino acids in root exudates remain stable under all the BaP stress treatments, albeit varying contents of the 19 types of amino acids under different $\mathrm{BaP}$ concentrations. The contents of all amino acid in root exudates increased with increasing $\mathrm{BaP}$ concentrations; especially, the contents of secreted threonine, serine, glycine and alanine increased significantly among the 19 types of amino acids and the differences were significant among all treatments with different BaP concentrations $(p<0.05)$. However, proline, hydroxyproline, and aspartic acid participated in the stress responses of plants almost in the form of additive or synergistic effects, and their contents in root exudates increased markedly with increasing $\mathrm{BaP}$ concentrations in soils; the differences among different treatments were significant $(p<0.05)$. The more constituents of amino acids there were in stress responses, the stronger the remediation potentials of plants would be. All these illustrate that the characteristics of root exudates in Festuca were closely related to their remediation potential under the BaP stress. The greater the remediation potentials were, the more organic compounds of low molecular weight there were and the more complex those compounds would be. Moreover, they also showed a stronger environment adaptability and physiological plasticity.

Key words benzo $[\alpha]$ pyrene; Festuca; root exudates; organic compounds of low molecule weight; interspecies difference

Citation: Pan SW, Yuan X, Liu C, Li YL, Yang T, Tang HY (2016). Effects of benzo $[\alpha]$ pyrene on the organic compounds of low molecule weight excreted by root systems in five Festuca species with different remediation potentials. Chinese Journal of Plant Ecology, 40, 604-614. doi: 10.17521/cjpe.2015.0426

多环芳烃 (polycyclic aromatic hydrocarbons, PAHs) 是环境中普遍存在的持久性有机污染物, 多 数具有致癌、致畸或致突变效应(Chen \& Liao, 2006)。因其性质稳定、水溶性差, 易于被土壤颗粒 吸附, 土壤中PAHs含量逐年上升(Tao et al., 2004; Li et al., 2006), 严重威胁着土壤环境质量及农产品 质量安全; 土壤中PAHs的迁移转化行为及其环境 效应已成为环境修复领域的研究热点(Mueller \& Shann, 2006; Lakshmanan et al., 2014)。

植物的存在能加快土壤中PAHs的去除(Joner \& Leyval, 2003; Kim et al., 2004; Gao et al., 2006), 根 系分泌物(root exudates)营造的根际微生态特征则 是影响PAHs快速消解的重要原因(Sumia et al., 2013;
Tejeda-Agredano et al., 2013)。植物修复过程中, 根 系分泌物在改善土壤属性、提高污染物的生物可利 用性、缓解环境胁迫等方面具有重要作用(Bertin \& Yang, 2003; Phillipsa et al., 2012; Shahzad et al., 2015)。一方面, 根系分泌的某些胞外酶能够直接参 与PAHs的降解过程; 另一方面, 分泌物中的可溶性 糖、低分子量有机酸、氨基酸等物质, 为根际微生 物提供充足的营养, 增加根际微生物的数量、提高 微生物活性, 进而加速 PAHs 降解(Susarla et al., 2002; Xie et al., 2012; Lakshmanan et al., 2014)。同时, 根系分泌物还会直接影响PAHs的固定和活化, 进 而影响PAHs在土壤-植物系统中的迁移转化行为、 促进PAHs去除(Marschner et al., 2004; Abhilash et al., 
2012); PAHs的环数越多, 根际效益越明显(Sun et al., 2010)。不同植物间, 因根系分泌物的组成成分 及其释放特征常存在一定差异, 对根际微生物的区 系结构、代谢活性的影响也不一样, 进而表现出不同 的修复潜力(Kirk et al., 2005; Luo et al., 2006; Hunter et al., 2014)。因此, 探讨PAHs胁迫下不同修复潜力植 物间根系分泌物中主要组分的释放特征及其种间差 异, 对于研究植物修复的生物学机制、揭示不同植物 间修复潜力的种间差异具有重要意义。鉴于此, 本研 究以对PAHs污染土壤具有一定修复潜力的羊茅属 (Festuca) 植物为试验对象, 以苯并 $[\alpha]$ 萠 (BaP) 为 PAHs代表物, 借助根际袋土培法, 对比研究PAHs 胁迫下不同修复潜力的5种羊茅属植物在不同生长 期时根系分泌物中几种低分子量有机物的释放特征, 为植物修复理论的研究与实践提供依据。

\section{1 材料和方法}

\section{1 试验材料}

\subsubsection{PAHs代表物}

$\mathrm{BaP}$ 是PAHs类污染物中分布最广、致癌性最强 的烃类物质, 其来源广泛、稳定性强, 且与其他 PAHs含量有一定相关性。本研究选择BaP (Aldrich, 纯度> 97\%)为PAHs代表物。

\subsection{2 供试植物}

供试植物为 5 种羊茅属植物: 苇状羊茅 $(F$. arundinacea, Fa)、草原羊茅(F. chelungkiangnica, Fc)、毛稃羊茅(F. rubra subsp. arctica, Fr)、贫芒羊 茅(F. sinomutica, Fm)、细芒羊茅(F. stapfii, Fs), 种 子购于四川省草原研究所。种子经 $3 \% \mathrm{H}_{2} \mathrm{O}_{2}$ 溶液消 毒20 min、蒸馏水冲洗干净后于烧杯中浸泡吸胀 24 $h$, 置于培养㿼中恒温 $\left(35{ }^{\circ} \mathrm{C}\right)$ 催芽 $15 \mathrm{~h}$ 后, 播种于根 际袋。

\subsection{3 供试土壤}

供试土壤为中性紫色土, 采自成都大学试验农 场表层土(5-20 cm, $44.6 \mathrm{pg} \cdot \mathrm{L}^{-1}$ 检出限下未检测到 $\mathrm{BaP})$ 。土壤理化性质: $\mathrm{pH} 7.19$, 有机质含量 $2.14 \times$ $10^{4} \mathrm{mg} \cdot \mathrm{kg}^{-1}$, 阳离子交换量(CEC) $20.43 \mathrm{cmol} \cdot \mathrm{kg}^{-1}$, 速效 $N 、 P 、 K$ 分别为31.62、24.74、94.81 $\mathrm{mg} \cdot \mathrm{kg}^{-1}$; 砂 粒、粉粒和黏粒的质量分数分别为 $71.27 \% 、 9.59 \%$ 和19.14\%。土壤风干后过2 mm篮, 备用。

\section{2 试验方法}

土培试验采用根际袋试验装置在智能温室内
进行(许超等, 2010), 涉及 3 个处理、 5 个水平(5个 BaP 胁迫水平下的 5 种植物、5 个生长期), 重复 3 次。具 体如下:

按照 $150 \mathrm{mg} \cdot \mathrm{kg}^{-1} \mathrm{CO}\left(\mathrm{NH}_{2}\right)_{2}$ 、 $50 \mathrm{mg} \cdot \mathrm{kg}^{-1}$ $\mathrm{Ca}\left(\mathrm{H}_{2} \mathrm{PO}_{4}\right) \cdot 2 \mathrm{H}_{2} \mathrm{O}$ 和 $100 \mathrm{mg} \cdot \mathrm{kg}^{-1} \mathrm{~K}_{2} \mathrm{SO}_{4}$ 比例补充底肥 后, 取适量土壤过 $2 \mathrm{~mm}$ 篎, 加入 $\mathrm{BaP}$ 丙酮溶液; 待 丙酮挥发后, 用未污染土壤不断稀释、搅拌, 过2 $\mathrm{mm}$ 篎、混匀, 结合农田土壤中PAHs的背景值, 获得 5 个污染水平。 $50 \%$ 田间持水量(WHC)、室温状态下 平衡7天(Pan et al., 2008; Wei \& Pan, 2010)后, 测得 土样中 $\mathrm{BaP}$ 的初始含量分别为: $10.25 \mathrm{mg} \cdot \mathrm{kg}^{-1}\left(\mathrm{~T}_{1}\right)$ 、 $20.37 \mathrm{mg} \cdot \mathrm{kg}^{-1}\left(\mathrm{~T}_{2}\right) 、 40.45 \mathrm{mg} \cdot \mathrm{kg}^{-1}\left(\mathrm{~T}_{3}\right) 、 80.24$ $\mathrm{mg} \cdot \mathrm{kg}^{-1}\left(\mathrm{~T}_{4}\right)$ 和161.74 $\mathrm{mg} \cdot \mathrm{kg}^{-1}\left(\mathrm{~T}_{5}\right)$ 。取污染土样 $2 \mathrm{~kg}$ 装入 $20 \mathrm{~cm} \times 15 \mathrm{~cm}$ 的塑料盆钵中, 其中的 $400 \mathrm{~g}$ 装 在300目尼龙网袋中; 尼龙网袋放在盆中央, 四周 及底部均为同质土样, 保持袋内外土壤高度相同。 每种污染水平分装75盆, 50\% WHC、室温状态下平 衡5天(Pan et al., 2008; Wei \& Pan, 2010); 选取籽粒 饱满、大小均匀的催芽后种子单播于袋内土壤中, 每种植物播种 15 盆, 每盆 10 颗, 置于温室中; 另设 一组没有播种的污染土样作为对照, 每个处理重复 3次。试验期间, 日间室温为 $25{ }^{\circ} \mathrm{C}$ 、夜间为 $18{ }^{\circ} \mathrm{C}$, 自 然光照条件; 每2天随机交换盆针在温室中的位置, WHC维持在 $50 \%$ (称重补水法)。播种日记为 0 天, 在 植物生长 $30 、 40 、 50 、 60$ 和70天时，分别对不同胁 迫强度的供试植物、土样破坏性取样: 连同根际袋 小心取出植物幼苗、尽可能不伤及其根系, 轻轻抖 落根际土壤后收集其根系分泌物; 混匀根际土壤、 非根际土壤后, 四分法取样, 装袋、冷冻干燥, 过 $1 \mathrm{~mm}$ 篮后低温 $\left(-20{ }^{\circ} \mathrm{C}\right)$ 保存、分析BaP残留量。

\section{3 根系分泌物收集}

取出根际袋, 用自来水将根洗净、去离子水冲 洗3遍后, 浸于盛有适量Milli-Q超纯水的烧杯中(锡 纸包裹杯壁, 确保根部避光; 液面高度以漫过根部 为宜), 自然光照下收集 $4 \mathrm{~h}$, 用少量高纯水冲洗根 系3次, 冲洗液与收集液合并、慢速滤过 $0.45 \mu \mathrm{m}$ 微 孔滤膜(Tu et al., 2004; 许超等, 2010)。将滤液定容 至 $50 \mathrm{~mL},-20{ }^{\circ} \mathrm{C}$ 以下储存, 待分析。取 $10 \mathrm{~mL}$ 滤液真 空冷冻干燥至干, 加 $1 \mathrm{~mL}$ Milli-Q超纯水溶解, -20 ${ }^{\circ} \mathrm{C}$ 以下储存, 用于分析低分子量有机酸; 取 $20 \mathrm{~mL}$ 滤液真空冷冻干燥至干, 加 $2 \mathrm{~mL}$ Milli-Q超纯水溶 解, $-20{ }^{\circ} \mathrm{C}$ 以下储存, 用于分析氨基酸(许超等, 
2010)。

\section{4 分析方法}

\subsection{1 可溶性糖测定}

使用葱酮比色法。可溶性糖含量以每kg根(干质 量)分泌的可溶性糖计量, 单位为 $\mathrm{mg} \cdot \mathrm{kg}^{-1}$ 。

\subsection{2 低分子量有机酸测定}

使用高效液相色谱(HPLC)测定。检测条件: Agilent 1100色谱仪(Agilent Technologies Palo Alto, USA), $X B-C_{18}$ 色谱柱、紫外检测器 $(\lambda=210 \mathrm{~nm})$; 流动 相为 $0.025 \mathrm{~mol} \cdot \mathrm{L}^{-1}$ 的 $\mathrm{H}_{3} \mathrm{PO}_{4}-\mathrm{KH}_{2} \mathrm{PO}_{4}$ 缓冲液 $(\mathrm{pH}$ 值 2.3 ), 流速为 $0.7 \mathrm{~mL} \cdot \mathrm{min}^{-1}$, 进样量为 $20 \mu \mathrm{L}$, 柱温 $30{ }^{\circ} \mathrm{C}$ 。所 用样品 3 个重复。低分子量有机酸释放量以每 $k g$ 根(干 质量)分泌的有机酸总量计量, 单位为 $\mathrm{mg} \cdot \mathrm{kg}^{-1}$ 。

\subsection{3 氨基酸测定}

使用日立L-8900型氨基酸自动分析仪(Hitachi, Tokyo, Japan)测定。检测条件: 离子交换柱规格2.6 $\mathrm{mm} \times 150 \mathrm{~mm}$, 交换树型号: NO 2619 (52051), 柱 温 $53{ }^{\circ} \mathrm{C}$, 泵流速 $0.225 \mathrm{~mL} \cdot \mathrm{min}^{-1}$, 进样量 $50 \mu \mathrm{L}$, 分 析时间 $72 \mathrm{~min}$ 。在检测波长 $\lambda=440 \mathrm{~nm}$ 条件下, 检出 限为 $2.2 \times 10^{-9} \mathrm{~mol}$, 保留时间重现性变异系数 $(\mathrm{CV})$ $1 \%$ 以下、峰面积重现性 $C V 1.5 \%$ 以下。所用样品 3 个重复。氨基酸量以每 $\mathrm{kg}$ 根(干质量)分泌的有机酸 总量计量, 单位为 $\mathrm{mg} \cdot \mathrm{kg}^{-1}$ 。

\subsubsection{BaP测定}

参照Pan等(2008)的方法, 略作改进: 取2.0 g土 样于离心管内, 加入 $2.0 \mathrm{~g}$ 无水 $\mathrm{Na}_{2} \mathrm{SO}_{4}$, 混匀; 加入

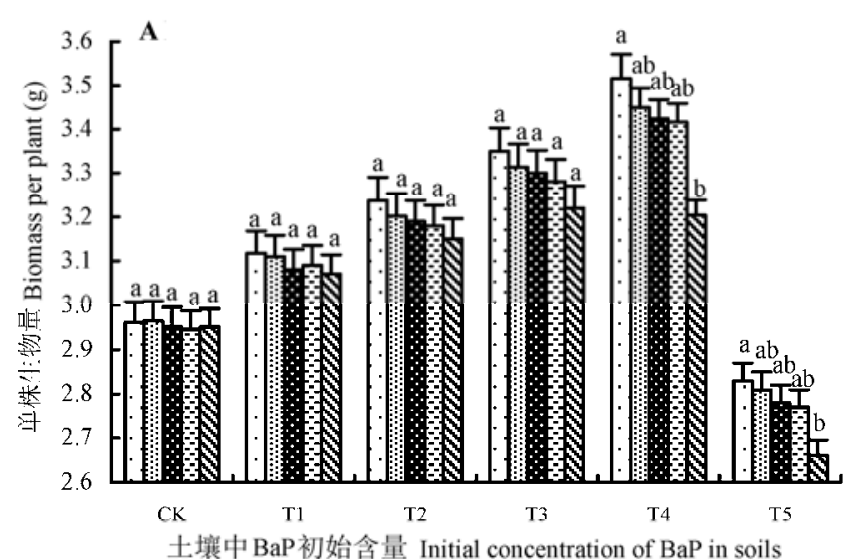

口苇状羊茅 Fa 回草原羊茅 Fc
$10 \mathrm{~mL}$ 二氯甲烷 $\left(\mathrm{CH}_{2} \mathrm{Cl}_{2}\right), 40{ }^{\circ} \mathrm{C}$ 下超声萃取 $1 \mathrm{~h}$; $4000 \mathrm{r} \cdot \mathrm{min}^{-1}$ 下离心。取 $3 \mathrm{~mL}$ 上清液过Fisher Pasteur 玻璃管硅胶柱, $1: 1$ 二氯甲烷和正己烷洗脱, $40{ }^{\circ} \mathrm{C}$ 下 浓缩至干, 用甲醇定容到 $2 \mathrm{~mL}$, 过 $0.22 \mu \mathrm{m}$ 微孔滤膜 后进行HPLC (Waters 600, Waters China, Hong Kong, China)分析。检测条件: DAD检测器, $\not 4.6 \times 250$ $\mathrm{mm} \mathrm{C}_{18}$ 反相色谱柱; 甲醇: 水 $(\mathrm{V} / \mathrm{V}, 83: 17)$ 为流动相, $\lambda=290 \mathrm{~nm}$ 。在此条件下, $\mathrm{BaP}$ 检测限为 $44.6 \mathrm{pg} \cdot \mathrm{L}^{-1}$, 加标回收率为 $94.36 \%(n=7$, 相对标准差 $(R S D)<$ $5.94 \%)$ )。

\section{5 数据分析}

数据经Microsoft Excel 2003处理后, 借助SPSS 11.5软件最小显著差异 $(L S D)$ 法分析不同处理间差 异显著性。

\section{2 结果和分析}

\section{$2.1 B a P$ 胁迫对待试植物生物量的影响}

图1为BaP胁迫下5种羊茅属植物在 50 天时的生 长状况。可以看出, 轻度 $\left(\mathrm{T}_{1}-\mathrm{T}_{2}\right)$ 或中度 $\left(\mathrm{T}_{3}-\mathrm{T}_{4}\right)$ 胁迫 刺激了植物生长, 5 种植物生物量(单株干质量)变化 呈现 $\mathrm{T}_{4} \geq \mathrm{T}_{3} \geq \mathrm{T}_{2} \geq \mathrm{T}_{1} \geq \mathrm{CK}$ 的趋势, $\mathrm{T}_{4}$ 水平时达到峰 值, 且 $\mathrm{T}_{4}>\mathrm{T}_{1} 、 \mathrm{~T}_{4}>C K(p<0.05)$; 细芒羊茅的生物 量变化虽然呈现 $\mathrm{T}_{4} \geq \mathrm{T}_{3} \geq \mathrm{T}_{2} \geq \mathrm{T}_{1} \geq \mathrm{CK}$ 的趋势, 但各 胁迫水平间差异不显著 $(p>0.05)$ 。重度 $\left(\mathrm{T}_{5}\right)$ 胁迫抑 制了植物生长, 其生物量明显低于其他胁迫水平 $(p$ $<0.05)$ 。植物的根冠比也呈现与生物量

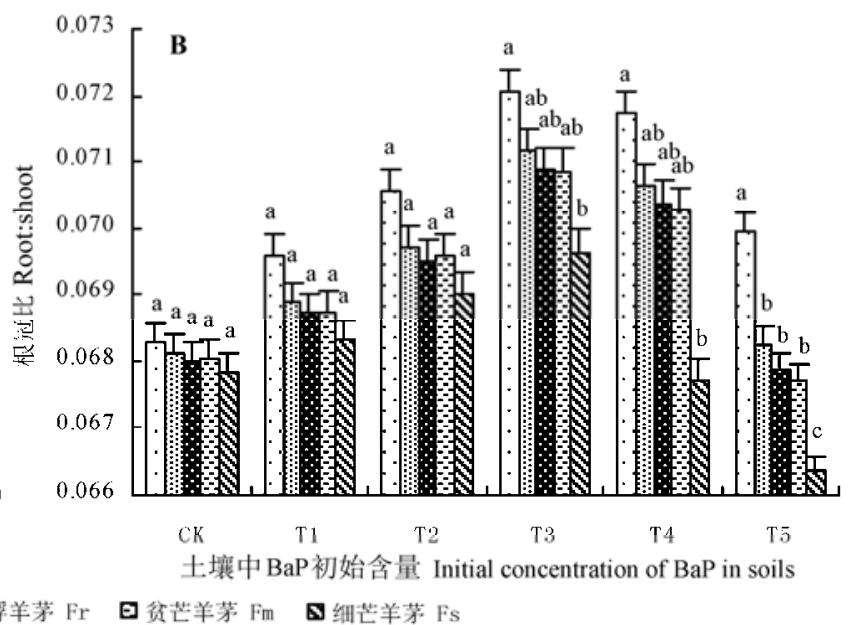

图1 苯并 $[\alpha]$ 芘 $(\mathrm{BaP})$ 胁迫对 5 种羊茅属植物生物量、根冠比的影响(平均值土标准误差)。 $C K 、 T_{1} 、 T_{2} 、 T_{3} 、 T_{4} 、 T_{5}, \mathrm{BaP}$ 的初始 含量分别为 $0 、 10.25 、 20.37 、 40.45 、 80.24$ 和 $161.74 \mathrm{mg} \cdot \mathrm{kg}^{-1}$ 。不同小写字母表示相同胁迫条件下差异显著 $(p<0.05)$ 。

Fig. 1 Biomass and root: shoot ratio of plants in five Festuca species growing in soils contaminated with different concentrations of benzo $[\alpha]$ pyrene $(\mathrm{BaP})$ (mean $\pm S E$ ). $\mathrm{CK}, \mathrm{T}_{1}, \mathrm{~T}_{2}, \mathrm{~T}_{3}, \mathrm{~T}_{4}$, and $\mathrm{T}_{5}$ designate the treatments with initial concentrations of BaP at 0 , 10.25 , 20.37, 40.45, 80.24 and $161.74 \mathrm{mg} \cdot \mathrm{kg}^{-1}$, respectively. Fa, F. arundinacea; Fc, F. chelungkiangnica; Fr, F. rubra subsp. arctica; Fm, F. sinomutica; Fs, F. stapfii. Different lowercase letters indicate significant differences $(p<0.05)$ under the same stress conditions. 
类似的变化, 但细芒羊茅的峰值 $(0.0695 \pm 0.0006)$ 出现在 $\mathrm{T}_{3}$ 水平, 之后则逐渐降低。相同胁迫强度下, 除 $\mathrm{T}_{4} 、 \mathrm{~T}_{5}$ 水平的细芒羊茅外, 5 种羊茅属植物的生物量 间无明显差异 $(p>0.05)$ 。总体上, BaP胁迫对细芒羊 茅的生长影响较大: 相同胁迫强度下, 其生物量、根 冠比都低于其他植物, 胁迫强度越大、趋势越明显; $\mathrm{T}_{3}-\mathrm{T}_{5}$ 水平时, 差异达到显著水平 $(p<0.05)$ 。

试验期间, $\mathrm{BaP}$ 胁迫下5种羊茅属植物在其他时 段的生长状态与 50 天时类似。总体上, 待试植物除 生物量略有变化外, 无其他表观受害症状(叶脉周 围出现褐色或黄褐色坏死斑点, 叶缘褪绿、叶尖干 枯, 叶片下垂; 叶色发黄、根毛减少), 均显示出较 强的BaP污染耐受能力。

\section{2 待试植物对BaP污染土壤修复潜力的种间差异}

图2为不同生长时期(30-70天)内5种羊茅属植 物对 $\mathrm{BaP}$ 污染土壤(10.25-161.74 $\mathrm{mg} \cdot \mathrm{kg}^{-1}$ )修复效果 的种间差异。可以看出, 植物的存在促进了土壤中 $\mathrm{BaP}$ 的去除: 相同污染水平下, 种植植物土壤中 $\mathrm{BaP}$ 的去除率远高于对照组 $(n=5, p<0.05)$, 修复期越 长, 差异越显著; 植物的种类不同, 对土壤中 $\mathrm{BaP}$ 的 去除效果也不一样。其间, 苇状羊茅表现出较强的 修复潜力, 草原羊茅、毛稃羊茅、贫芒羊茅次之, 细芒羊茅最差。相同污染条件下, 5 种羊茅属植物对 $\mathrm{BaP}$ 污染土壤的修复潜力依次为: 苇状羊茅 $>$ 草原 羊茅 $\geq$ 毛稃羊茅 $\geq$ 贫芒羊茅> 细芒羊茅, 但草原羊
茅与毛稃羊茅间、毛稃羊茅和贫芒羊茅间差异不 甚显著 $(p>0.05)$ 。

不同修复期(生长期)内, 植物的修复潜力也不 一样。修复前期(30-40天)、后期(60-70天)均低于修 复中期(40-60天) $(p<0.05)$, 呈现明显出“S”形变化 曲线，曲线拐点出现在修复期的第50天前后。

\section{3 待试植物根系分泌物中几种低分子量组分的}

\section{释放特征}

\subsection{1 可溶性糖的释放特征}

图3显示, $\mathrm{BaP}$ 胁迫不同程度地促进了根系分泌 物中可溶性糖的分泌, 并随着胁迫强度增大(图 3A)、胁迫期延长(图3B)呈现“先升后降”变化。相同 胁迫期(图3A：50天)内, 各处理组根系可溶性糖的 分泌量均高于对照组(CK), $\mathrm{T}_{3}$ 胁迫水平下达到峰值; $T_{2} 、 T_{3} 、 T_{4}$ 处理与CK组间差异显著 $(p<0.05)$, 但 $T_{1}$ 、 $\mathrm{T}_{5}$ 处理与 $\mathrm{CK}$ 组间差异不显著 $(p>0.05)$ 。相同胁迫水 平(图3B: $\mathrm{T}_{3}$ )下，不同胁迫期内根系可溶性糖分泌 量也不一样, 40 天时最多, 且明显多于其他时期 $(p<$ 0.05), 但在30、60和70天时可溶性糖释放量差异不 大 $(p>0.05)$ 。可见, $\mathrm{BaP}$ 胁迫能促进植物根系分泌更 多的可溶性糖, 在一定胁迫强度、胁迫期内, 可溶性 糖分泌量随胁迫强度的增强而增多; 超越此范围, 分泌量开始减少。

\subsection{2 低分子量有机酸的释放特征}

5 种羊茅属植物根系分泌物中, 草酸 $(83.76 \% \pm$

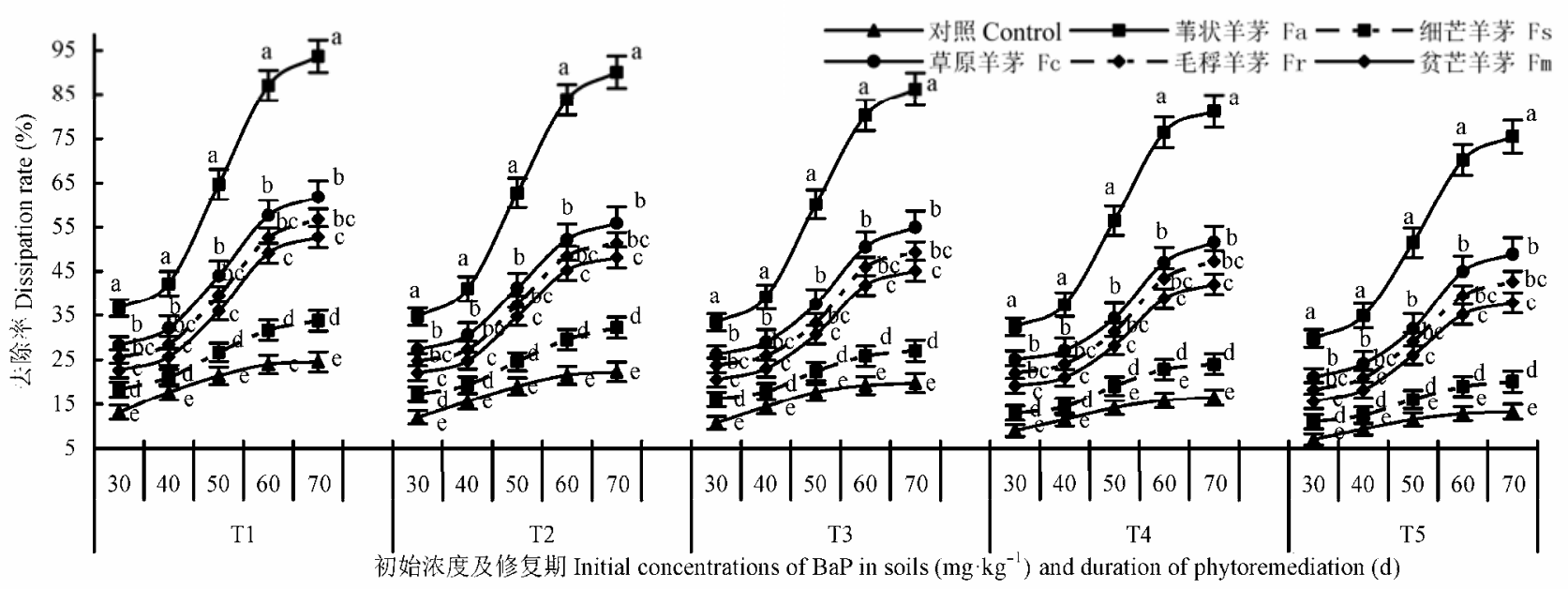

图2 待试植物对 BaP污染土壤修复潜力的种间差异(平均值士标准误差)。 $T_{1} 、 T_{2} 、 T_{3} 、 T_{4} 、 T_{5}, B a P$ 的初始含量分别为 10.25 、 20.37、40.45、80.24和161.74 $\mathrm{mg} \cdot \mathrm{kg}^{-1}$ 。数据源右侧的不同小写字母表示相同胁迫条件下差异显著 $(p<0.05)$ 。

Fig. 2 Differences in phytoremediation potentials of benzo $[\alpha]$ pyrene $(\mathrm{BaP})$-contaminated soils among five Festuca species (mean $\pm S E$ ). $\mathrm{T}_{1}, \mathrm{~T}_{2}, \mathrm{~T}_{3}, \mathrm{~T}_{4}$, and $\mathrm{T}_{5}$ designate the treatments with initial concentrations of $\mathrm{BaP}$ at 10.25, 20.37, 40.45, 80.24 and 161.74 $\mathrm{mg} \cdot \mathrm{kg}^{-1}$, respectively. Fa, F. arundinacea; Fc, F. chelungkiangnica; Fr, F. rubra subsp. arctica; Fm, F. sinomutica; Fs, F. stapfii. Different lowercase letters next to the right of data points indicate significant differences $(p<0.05)$ under the same stress conditions. 

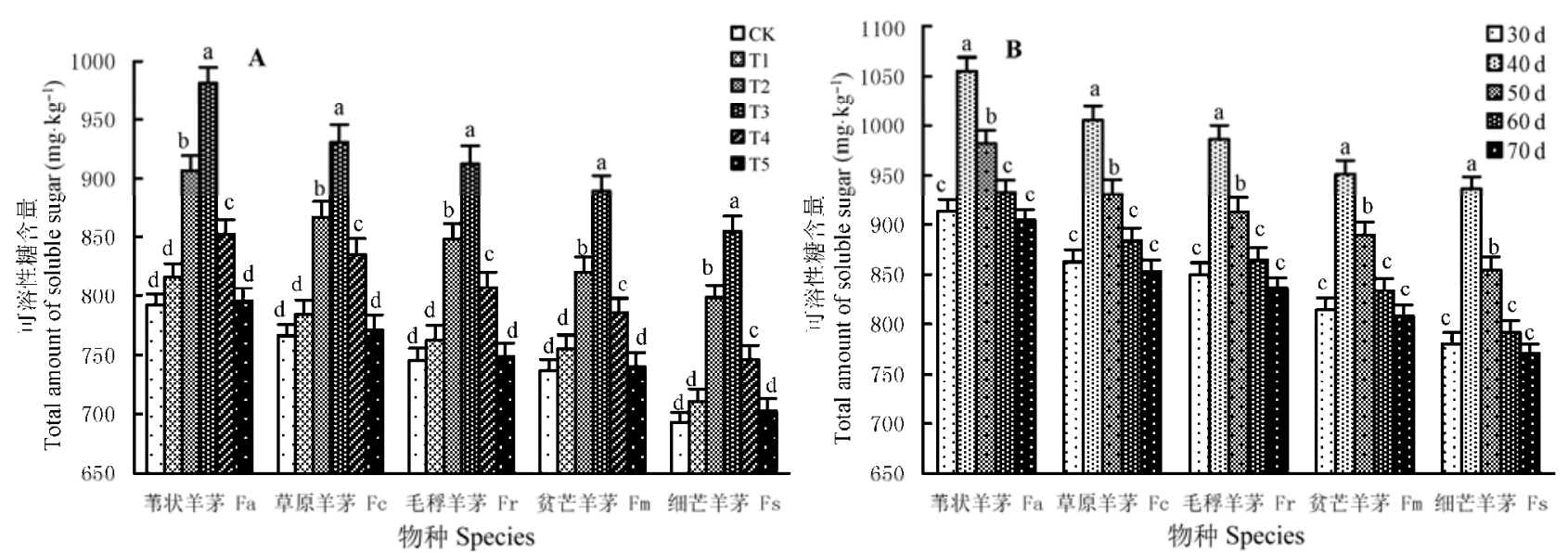

图3 苯并 $[\alpha]$ 芘 $(\mathrm{BaP})$ 的胁迫强度 $(\mathbf{A}) 、 \mathrm{~T}_{3}$ 处理下胁迫期(B)对 5 种羊茅属植物根系分泌物中可溶性糖含量的影响(平均值土标准误 差)。CK、 $T_{1} 、 T_{2} 、 T_{3} 、 T_{4} 、 T_{5}, B a P$ 的初始含量分别为 $0 、 10.25 、 20.37 、 40.45 、 80.24$ 和 $161.74 \mathrm{mg} \cdot \mathrm{kg}^{-1}$ 。不同小写字母表示 相同胁迫条件下差异显著 $(p<0.05)$ 。

Fig. 3 The effects of benzo $[\alpha]$ pyrene (BaP) stress level (A) and stress stage under $T_{3}$ treatment $(\mathbf{B})$ on the total amount of soluble sugars in root exudates of five Festuca species (mean $\pm S E$ ) . CK, $\mathrm{T}_{1}, \mathrm{~T}_{2}, \mathrm{~T}_{3}, \mathrm{~T}_{4}$, and $\mathrm{T}_{5}$ designate the treatments with initial concentrations of BaP at 0, 10.25, 20.37, 40.45, 80.24 and $161.74 \mathrm{mg} \cdot \mathrm{kg}^{-1}$, respectively. Fa, F. arundinacea; Fc, F. chelungkiangnica; Fr, $F$. rubra subsp. arctica; Fm, F. sinomutica; Fs, F. stapfii. Different lowercase letters indicate significant differences $(p<0.05)$ under the same stress conditions.

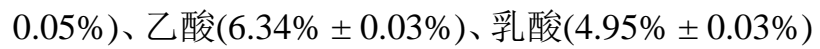
和苹果酸 $(2.29 \% \pm 0.02 \%)$ 的质量分数之和超过了低 分子量有机酸总量的 $97.34 \%$ 。苇状羊茅的根系分泌 物中检测出微量的顺丁烯二酸 $(0.27 \% \pm 0.01 \%)$ 、反 丁烯二酸 $(0.18 \% \pm 0.01 \%)$, 草原羊茅、毛稃羊茅、 贫芒羊茅的根系分泌物中检测出反顺丁烯二酸 $(0.43 \% \pm 0.02 \%)$, 细芒羊茅的根系分泌物中仅检测 出微量顺丁烯二酸 $(0.16 \% \pm 0.01 \%)$; 不同污染水平 下同种植物根系分泌物中低分子量有机酸的组成成 分基本不变。图4为待试植物根系在不同胁迫期内释 放的低分子量有机酸总量的变化趋势。

图4A显示, 相同胁迫期(50天)内, 苇状羊茅根 系分泌的有机酸总高于相同污染水平下的其他植物, 污染水平越高、有机酸分泌量越多, 呈现 $\mathrm{T}_{5} \geq \mathrm{T}_{4}>\mathrm{T}_{3}$ $>\mathrm{T}_{2}>\mathrm{T}_{1}>\mathrm{CK}$ 趋势; 除 $\mathrm{T}_{4}$ 与 $\mathrm{T}_{5}$ 间外, 各污染水平间 差异显著 $(p<0.05)$ 。草原羊茅、毛稃羊茅、贫芒羊 茅根系分泌的有机酸总量以 $\mathrm{T}_{4}$ 水平最高, 呈现 $\mathrm{T}_{4}>$ $\mathrm{T}_{3} \geq \mathrm{T}_{5}>\mathrm{T}_{2}>\mathrm{T}_{1}>C K$ 趋势; 除 $\mathrm{T}_{3}$ 与 $\mathrm{T}_{5}$ 间外, 各污染 水平间差异显著 $(p<0.05)$ 。细芒羊茅以 $\mathrm{T}_{3}$ 水平最高, 呈现 $\mathrm{T}_{3}>\mathrm{T}_{4} \geq \mathrm{T}_{2}>\mathrm{T}_{5} \geq \mathrm{T}_{1}>C$ K趋势, 但 $\mathrm{T}_{4}$ 与 $\mathrm{T}_{2} 、 \mathrm{~T}_{5}$ 与 $\mathrm{T}_{1}$ 间差异不显著 $(p>0.05)$ 。

图4B显示, 相同胁迫水平 $\left(\mathrm{T}_{3}\right)$ 下, 植物根系在 不同胁迫期释放的有机酸总量也不一样。修复潜力 最强的苇状羊茅在 30 天时释放量最多, 呈现 30 天> 40 天 $>50$ 天 $>60$ 天 $\geq 70$ 天趋势; 除第 $60 、 70$ 天外, 其
余各时期间差异显著 $(p<0.05)$ 。修复潜力较强的草 原羊茅、毛稃羊茅、贫芒羊茅在 30 天时的释放量最 多 $(p<0.05)$, 其余各时期间差异不大 $(p>0.05)$; 修 复潜力最差的细芒羊茅在各个时期的释放量无明显 差异 $(p>0.05)$ 。

\subsection{3 氨基酸的释放特征}

除色氨酸外, 其他19种常见氨基酸均能在待试 植物的根系分泌物中检测到。总体上, BaP胁迫促进 了氨基酸的分泌, 胁迫强度越大、分泌量越多。相 同胁迫期(50天; 图5A)内, 苇状羊茅根系的分泌量 总高于相同污染水平下的其他植物 $(p<0.05)$, 不同 污染水平间差异显著 $(p<0.05)$; 草原羊茅、毛稃羊 茅、贫芒羊茅根系的分泌量呈现 $\mathrm{T}_{5} \geq \mathrm{T}_{4}>\mathrm{T}_{3}>\mathrm{T}_{2}>$ $\mathrm{T}_{1}>\mathrm{CK}$ 趋势, $\mathrm{T}_{5} 、 \mathrm{~T}_{4}$ 间差异不显著 $(p>0.05)$; 细芒羊 茅根系的分泌量虽随着污染水平的升高而增多, 但 $\mathrm{T}_{5} 、 \mathrm{~T}_{4} 、 \mathrm{~T}_{3}$ 间差异不显著 $(p>0.05)$ 。相同胁迫强度 $\left(\mathrm{T}_{3}\right.$; 图5B)下, 苇状羊茅根系的分泌量总多于其他 植物 $(p<0.05), 30$ 天时的分泌量明显低于其他各时 期 $(p<0.05)$; 自 40 天起趋于稳定, 各时期释放量无 显著差异 $(p>0.05)$ 。草原羊茅、毛稃羊茅、贫芒 羊茅根系的释放量呈现 30 天 $<40$ 天 $<50$ 天 $\leq 60$ 天 $\leq 70$ 天趋势，30、40、50天间差异显著 $(p<0.05)$; 自50 天起趋于稳定, 各时期释放量差异不大 $(p>0.05)$ 。 细芒羊茅根系的释放量虽随胁迫时期的延长而增多, 但60、70天时的差异不显著 $(p>0.05)$ 。 


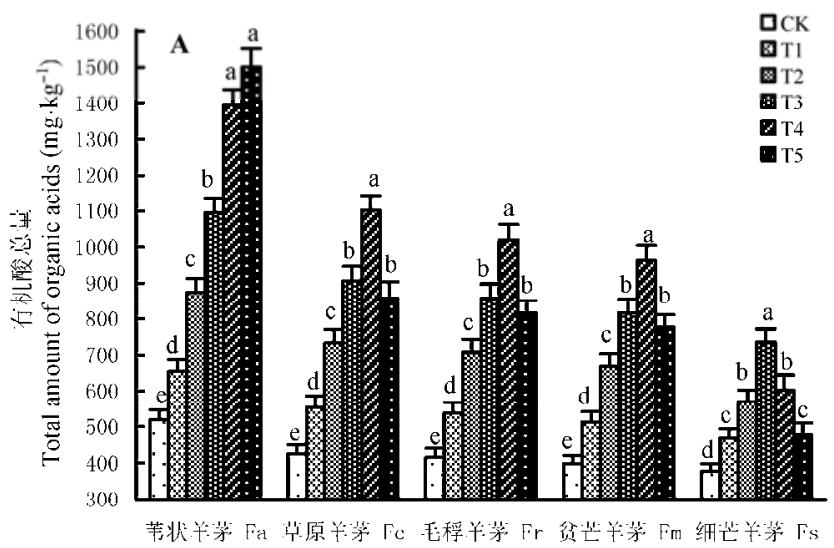

待试植物 Plant species tested

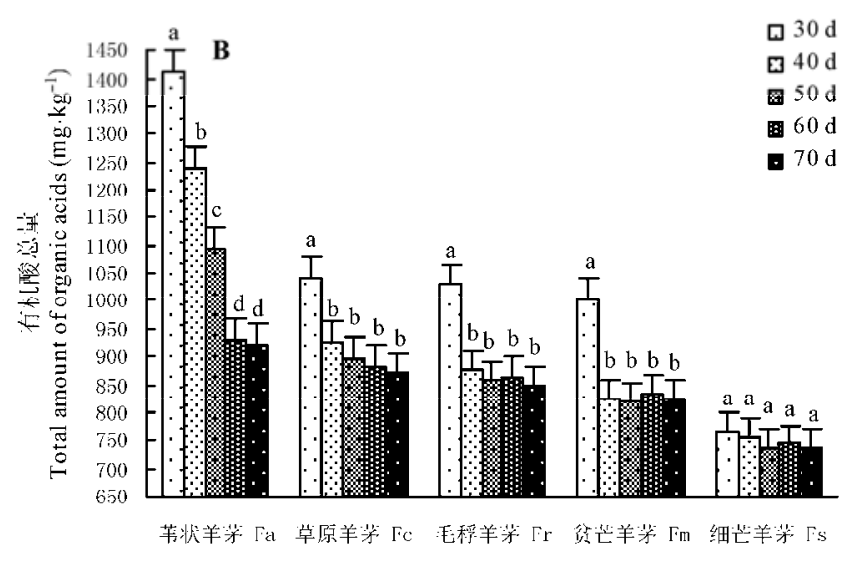

待试㥀物 Plant species tested

图4 苯并 $[\alpha]$ 萠 $(\mathrm{BaP})$ 的胁迫强度 $(\mathrm{A}) 、 \mathrm{~T}_{3}$ 处理下胁迫期(B)对 5 种羊茅属植物根系分泌物中有机酸总量的影响(平均值土标准误 差)。CK、 $T_{1} 、 T_{2} 、 T_{3} 、 T_{4} 、 T_{5}, B a P$ 的初始含量分别为 $0 、 10.25 、 20.37 、 40.45 、 80.24$ 和 $161.74 \mathrm{mg} \cdot \mathrm{kg}^{-1}$ 。不同小写字母表示 相同胁迫条件下差异显著 $(p<0.05)$ 。

Fig. 4 The effects of benzo $[\alpha]$ pyrene (BaP) stress level $(\mathbf{A})$ and stress stage under $\mathrm{T}_{3}$ treatment $(\mathbf{B})$ on the total amount of organic acids in root exudates of five Festuca species (mean $\pm S E$ ). . CK, $\mathrm{T}_{1}, \mathrm{~T}_{2}, \mathrm{~T}_{3}, \mathrm{~T}_{4}$, and $\mathrm{T}_{5}$ designate the treatments with initial concentrations of BaP at 0, 10.25, 20.37, 40.45, 80.24 and $161.74 \mathrm{mg} \cdot \mathrm{kg}^{-1}$, respectively. Fa, F. arundinacea; Fc, F. chelungkiangnica; Fr, F. rubra subsp. arctica; Fm, F. sinomutica; Fs, F. stapfii. Different lowercase letters indicate significant differences $(p<0.05)$ under the same stress conditions.
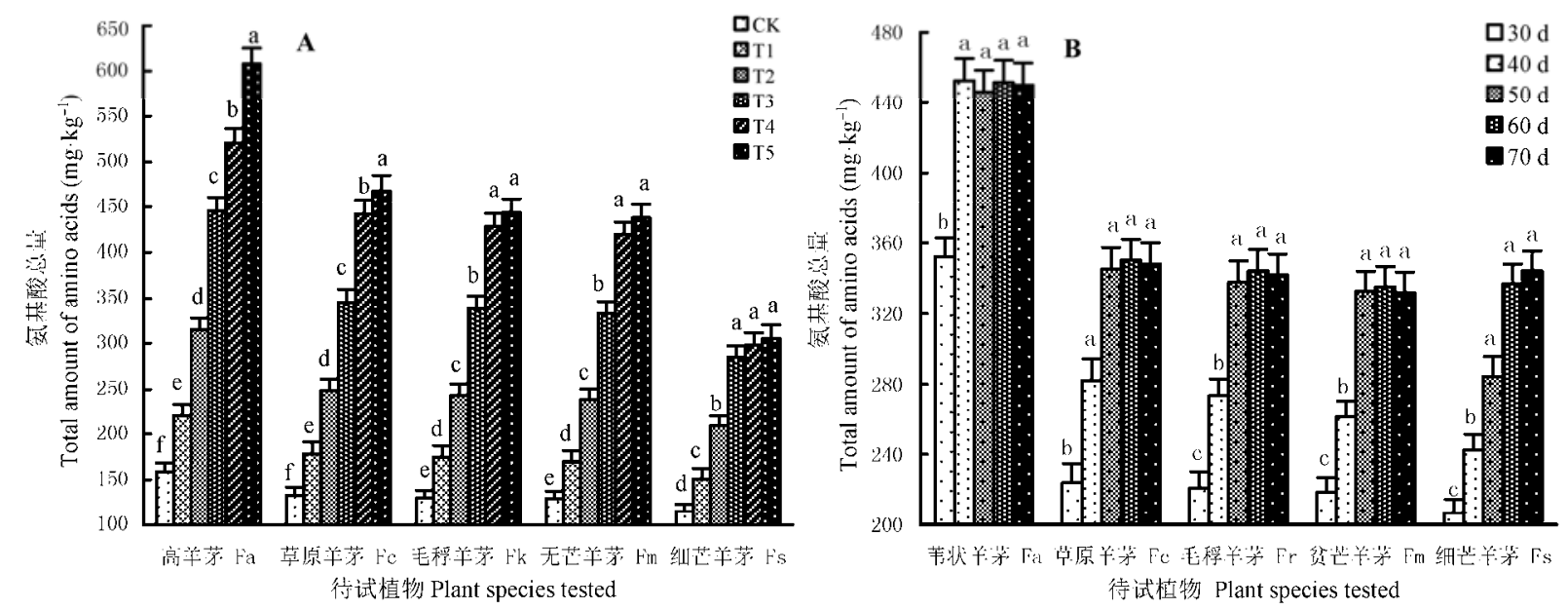

图5 苯并 $[\alpha]$ 萠 $(\mathrm{BaP})$ 的胁迫强度 $(\mathrm{A}) 、 \mathrm{~T}_{3}$ 处理下胁迫期(B)对 5 种羊茅属植物根系分泌物中氨基酸总量的影响(平均值土标准误 差)。CK、 $T_{1} 、 T_{2} 、 T_{3} 、 T_{4} 、 T_{5}, B a P$ 的初始含量分别为 $0 、 10.25 、 20.37 、 40.45 、 80.24$ 和161.74 mg $\mathrm{kg}^{-1}$ 。不同小写字母表示 相同胁迫条件下差异显著 $(p<0.05)$ 。

Fig. 5 The effects of benzo $[\alpha]$ pyrene $(\mathrm{BaP})$ stress level $(\mathbf{A})$ and stress stage under $\mathrm{T}_{3}$ treatment $(\mathbf{B})$ on the total amount of amino acids in root exudates of five Festuca species (mean $\pm S E$ ). $C K, \mathrm{~T}_{1}, \mathrm{~T}_{2}, \mathrm{~T}_{3}, \mathrm{~T}_{4}$, and $\mathrm{T}_{5}$ designate the treatments with initial concentrations of $\mathrm{BaP}$ at $0,10.25,20.37,40.45,80.24$ and $161.74 \mathrm{mg} \cdot \mathrm{kg}^{-1}$, respectively. Fa, F. arundinacea; Fc, F. chelungkiangnica; Fr, $F$. rubra subsp. arctica; Fm, F. sinomutica; Fs, F. stapfii. Different lowercase letters indicate significant differences $(p<0.05)$ under the same stress conditions.

试验期间，BaP胁迫对根系分泌物中氨基酸的 种类影响不大, 但对分泌量的影响较大, 且影响程 度大小不一。相比之下, 苏氨酸、丝氨酸、甘氨酸、 丙氨酸、脯氨酸和羟脯氨酸的释放特征较为突出。

与CK组相比, $\mathrm{BaP}$ 胁迫促进了植物根系对苏氨 酸、丝氨酸、甘氨酸、丙氨酸的释放活动。试验浓 度 $\left(\mathrm{T}_{1}-\mathrm{T}_{5}\right)$ 范围内, 其分泌量随着胁迫强度的增大而
剧增, 不同胁迫水平间差异显著 $(p<0.05)$; 脯氨 酸、羟脯氨酸和天冬氨酸在苇状羊茅根系也呈现类 似的释放特征, 但在细芒羊茅根系的释放量却忽高 忽低、多少不一(表1)。其间，草原羊茅根系分泌的 脯氨酸和天冬氨酸、毛稃羊茅根系分泌的脯氨酸和 着弪脯氨酸、贫芒羊茅根系分泌的天冬氨酸均随着 $\mathrm{BaP}$ 胁迫强度的增大而剧增, 不同水平间差异显著 
表1 苯并 $[\alpha]$ 萠 $(\mathrm{BaP})$ 胁迫 50 天时 5 种羊茅属植物根系分泌物中 7 种氨基酸释放量 $\left(\mathrm{mg} \cdot \mathrm{kg}^{-1}\right)$

Table 1 Amount of seven amino acids released in root exudates of five Festuca species tested on day 50 under benzo $[\alpha]$ pyrene $(B a P)$ stress $\left(\mathrm{mg} \cdot \mathrm{kg}^{-1}\right.$ )

\begin{tabular}{|c|c|c|c|c|c|c|}
\hline 氨基酸 & Acids & 苇状羊茅 $\mathrm{Fa}$ & 草原羊茅 Fc & 毛稃羊茅 Fr & 贫芒羊茅 Fm & 细芒羊茅 Fs \\
\hline 苏氨酸 & Thr & $7.28,21.29(10.31-31.53)^{\uparrow^{*}}$ & $6.74,16.81(8.42-26.35)^{\uparrow^{*}}$ & $7.12,17.36(9.24-26.39)^{\uparrow^{*}}$ & $6.52,18.81(8.78-29.03)^{\uparrow^{*}}$ & $4.55,12.18(6.36-20.95)^{\uparrow^{*}}$ \\
\hline 丝氨酸 & Ser & 29.36, $79.94(40.95-116.42)^{\uparrow *}$ & $22.24,60.12(34.25-81.27)^{\uparrow^{*}}$ & $22.07,65.74(38.42-76.05)^{\uparrow *}$ & $19.08,42.11(27.24-64.31)^{\uparrow^{*}}$ & $19.60,44.27(25.63-62.48)^{\uparrow *}$ \\
\hline 甘氨酸 & Gly & $3.72,11.62(5.43-18.11)^{\uparrow^{*}}$ & $3.17,8.84(4.47-15.91)^{\uparrow^{*}}$ & 2.62, $9.56(3.93-16.07)^{\uparrow^{*}}$ & 2.72, $9.51(4.11-16.42)^{\uparrow^{*}}$ & 2.12, $6.75(3.35-12.67)^{\uparrow^{*}}$ \\
\hline 丙氨酸 & Ala & $5.63,17.09(8.16-26.14)^{\uparrow^{*}}$ & $4.57,14.79(6.94-24.07)^{\uparrow^{*}}$ & $4.09,14.91(5.81-21.47)^{\uparrow^{*}}$ & $3.95,10.17(5.74-22.41)^{\uparrow^{*}}$ & $3.42,10.98(5.14-19.35)^{\uparrow^{*}}$ \\
\hline 脯氨酸 & Pro & $10.73,31.45(16.73-47.24)^{\uparrow^{*}}$ & 8.56, $25.33(12.12-39.56)^{\uparrow^{*}}$ & 6.64, $21.37(8.42-33.07)^{\uparrow^{*}}$ & $7.82,18.17(8.57-28.44)^{\uparrow}$ & $6.90,12.78(4.28-20.07)^{\uparrow \downarrow}$ \\
\hline 羟脯氨酌 & 酸 Hyp & $12.50,35.42(17.87-52.94)^{\uparrow^{*}}$ & $11.06,11.57(11.34-11.72)^{\uparrow}$ & $8.16,27.08(12.84-46.87)^{\uparrow^{*}}$ & $9.21,16.74(9.91-31.17)^{\uparrow}$ & $8.10,15.97(7.49-20.12)^{\uparrow \downarrow}$ \\
\hline 天冬氨 & 酸 Asp & $14.20,39.36(20.08-58.62)^{\uparrow^{*}}$ & $9.86,18.87(12.07-31.48)^{\uparrow^{*}}$ & $8.77,9.65(9.02-10.13)^{\uparrow}$ & $8.15,24.32(11.47-38.25)^{\uparrow^{*}}$ & $9.27,17.07(8.24-26.17)^{\uparrow \downarrow}$ \\
\hline
\end{tabular}

表中数据“A, B (C-D)”所表示的氨基酸释放量分别为对照组含量(A)、 BaP处理的平均值(B)、最低值(C)及最高值(D)。 $\uparrow *$ 表示该氨基酸释放量随着土壤 中 $\mathrm{BaP}$ 胁迫强度的增大而剧增, 不同处理间差异显著 $(p<0.05)$; $\uparrow$ 表示该氨基酸释放量随着 $\mathrm{BaP}$ 胁迫强度的增大而增加, 不同处理间差异不显著 $(p>$ $0.05) ; \uparrow \downarrow$ 表示该氨基酸释放量与 BaP胁迫强度无明显相关性。

The data “A, B (C-D)” in each cell of this table indicate the amount of amino acids in root exudates in control (A), and the average (B), minimum (C) and maximum (D) among of amino acids under BaP treatments, respectively. $\uparrow *$ indicate that the amount of amino acids released in root exudates markedly increased with increasing $\mathrm{BaP}$ concentrations in soils, and the differences were significant among treatments $(p<0.05)$; $\uparrow$ indicate that the amount of amino acids released increased with increasing BaP concentrations, but the differences were not significant among treatments $(p>0.05)$; $\uparrow \downarrow$ indicate that the amount of amino acids released in root exudates has no apparent correlation with the concentrations of BaP in soils. Fa, F. arundinacea; Fc, F. chelungkiangnica; Fr, F. rubra subsp. arctica; Fm, F. sinomutica; Fs, F. stapfii.

$(p<0.05)$; 但草原羊茅根系分泌的羟脯氨酸、毛稃 羊茅根系分泌的天冬氨酸, 以及贫芒羊茅根系分 泌的脯氨酸、羟脯氨酸虽随着胁迫强度的增大而增 多, 但不同水平间差异不显著 $(p>0.05)$ (表1)。

\section{3 讨论}

植物根际对PAHs的降解效果与修复植物的种 类有关。Yi和Crowley (2007) 研究43种植物根部组织 对PAHs的降解作用发现, 有些植物对PAHs降解有 明显的促进作用, 有些则无作用或有抑制作用。根 系分泌物所营造的根际环境及其所呈现的根际效应 是植物响应外界胁迫的重要途径, 也是影响修复效 果的关键因素(Gao et al., 2010; 吴林坤等, 2014)。相 同胁迫条件下，根系分泌物的释放特征及其主要组 分的种间差异主要处决于植物自身基因型的特异性 及其生理状态的个体差异(Zhu et al., 2009; D’Orazio et al., 2013)。本研究以亲缘关系较近的5种羊茅属植 物为实验对象, 其基因型差异相对较小, 在一定程 度上能增加研究结果的可比性。尽管待试植物在无 污染 $(C K)$ 、轻度或中度污染 $\left(\mathrm{T}_{1}-\mathrm{T}_{4}\right)$ 时的生物量, 以 及被植物积累的 $\mathrm{BaP}$ 量 $(0.08 \%-0.21 \%$, 平均 $(0.15 \pm$ 0.02)\%差异甚微, 但它们对 $\mathrm{BaP}$ 污染土壤的修复潜 力却存在显著的种间差异。可见, 植物修复过程中, 即使是遗传关系很近的同属植物间, 修复潜力的种 间差异仍然存在。

植物生长指标的变化是植物受到不同程度胁
迫伤害的直观表现症状(王海翠等, 2013)。本试验中, 低度 $\left(\mathrm{T}_{1}-\mathrm{T}_{2}\right)$ 或中度 $\left(\mathrm{T}_{3}-\mathrm{T}_{4}\right) \mathrm{BaP}$ 胁迫刺激了植物生 长, $\mathrm{T}_{4}$ 水平时生物量出现峰值, 重度 $\left(\mathrm{T}_{5}\right)$ 胁迫则抑制 了植物生长。通常, 多数植物对PAHs 类环境胁迫具 有一定的耐受域值, 而这种域值与PAHs的理化性 质、植物种类和环境状况(Howsam et al., 2000; Line et al., 2003; 田晓雪等, 2008)以及PAHs进入细胞后 的累积或转化机制等因素有关(刘玉和周璐璐, 2011; 刘静等, 2015)。一定域值内, 植物生长受到的抑制 影响并不显著, 甚至能刺激植物生长; 超过这一域 值, 植物生长就会受到抑制。Maliszewska-Kordybach和Smreczak (2000)报道, 由芴、葱、萠和䓛 4 种 PAHs组成的混合物, 在浓度不超过 $10 \mathrm{mg} \cdot \mathrm{kg}^{-1}$ 时促 进了小麦(Triticum aestivum)、玉米(Zea mays)及燕麦 (Avena sativa)的生长, 高浓度时抑制生长; 刘泓等 (2008) 研究了不同浓度的荧葱培养基对拟南芥 (Arabidopsis thaliana)生长的影响, 发现不同浓度的 苂葱胁迫30天后拟南芥生长量明显受到不同程度的 抑制。尽管这些研究都表明, 高浓度PAHs胁迫抑制 植物的生长，但抑制作用机制尚不明确; 低浓度的 PAHs 对植物生长的促进作用, 有学者推测是因为 PAHs 具有与植物生长激素类似的作用(陈世军等, 2012), 但也仅仅局限于推测, 还没有被试验和实践 证实。

在土壤中, 根系分泌物与根际微生物间的相互 作用是一个十分重要的过程: 根系通过分泌各种次 
生代谢物质对根际微生物的种类、数量和分布产生 影响, 对根际微生物群落结构有选择塑造作用, 进 而影响PAHs的降解(Abhilash et al., 2012; Edwards et al., 2015)。本研究显示, 胁迫环境中植物根系可溶 性糖、低分子量有机酸、氨基酸等组分的释放特征 与植物自身的修复潜力有关: 修复潜力较强的苇状 羊茅所分泌的可溶性糖、低分子量有机酸、氨基酸 总多于其他几种植物; 修复潜力越强, 其分泌量、 组成成分的变化幅度越大。相同胁迫条件下, 修复 潜力较强的植物对 $\mathrm{BaP}$ 的胁迫响应总表现出更强的 环境适应性、生理活性上的可塑性, 修复潜力越强, 趋势越明显(图3-5)。可以推测, 根系分泌物的释放 特征势必影响根际微生物群落结构, 并产生选择性 塑造作用, 但这种释放特征是植物为减轻 $\mathrm{BaP}$ 毒害 而进行的自我生理调节还是 $\mathrm{BaP}$ 毒害破坏了根系正 常的生理活动而造成细胞内有机物质的渗漏尚待进 一步研究。

环境胁迫可能会影响根系分泌物的种类和数 量(沈宏等, 2002; Wu et al., 2014)。本试验在修复潜 力较强植物的根系分泌物中检测到一种低分子量有 机酸一反丁烯二酸, 它可能与植物对 $\mathrm{BaP}$ 类有害 物质的应激反应有关: 植物在应激反应过程中所产 生的破坏性氧自由基(reactive oxygen species, ROS) 容易与细胞内的生物大分子发生反应，对线粒体、 核糖体、溶酶体、过氧化物体、微粒体等细胞器以 及核酸、细胞骨架蛋白等生物大分子的结构和功能 产生损伤或毒害作用(Ramos \& Garcia, 2007), 反丁 烯二酸类抗氧化助剂对活性氧自由基较敏感, 能及 时捕获并清除胁迫过程中产生的各种活性氧自由 基、缓解环境胁迫的损害。但为什么仅在修复潜力 极强的苇状羊茅、极差的细芒羊茅的根系分泌物中 检测到顺丁烯二酸，尚待进一步研究。

根系分泌物的释放特征是植物根系胁迫反应 时的集中表现(Jennifer et al., 2015)。BaP胁迫促进了 5 种羊茅属植物对苏氨酸、丝氨酸、甘氨酸、丙氨酸 的释放, 许超等(2010)、谢晓梅等(2011)等在研究萠 胁迫对玉米、黑麦草根系分泌物的影响时也得出类 似结论。本试验中, 根系分泌物中脯氨酸、羟脯氨 酸和天冬氨酸等组分对 $\mathrm{BaP}$ 的胁迫响应更近乎以加 和效应(additivity)甚至协同效应(synergism)形式参 与植物的修复过程, 参与应激反应的组分越多, 修 复潜力越强: 修复潜力最强的苇状羊茅根系以上 3
种组分的释放量均随胁迫强度的增强而剧增; 修复 潜力次强的草原羊茅、毛稃羊茅根系上述组分中某 2 种的释放量随胁迫强度的增强而剧增; 修复潜力 次差的贫芒羊茅根系仅上述组分中某1种的释放量 随胁迫强度的增强而剧增; 修复潜力最差的细芒羊 茅根系上述组分的释放量与 $\mathrm{BaP}$ 胁迫强度无明显相 关性。但该响应模式是否具有普遍性, 尚待进一步 验证。

\section{4 结论}

(1)试验浓度范围内, 待试植物对 $\mathrm{BaP}$ 胁迫具有 较强的耐受能力, 其修复潜力依次为: 苇状羊茅> 草原羊茅 $\geq$ 毛稃羊茅 $\geq$ 贫芒羊茅 $>$ 细芒羊茅, 但草 原羊茅与毛稃羊茅、毛稃羊茅与贫芒羊茅间差异 不显著 $(p>0.05)$ 。

(2) $\mathrm{BaP}$ 胁迫促进了植物根系对可溶性糖的分 泌, 呈现“先升后降”波动: 一定胁迫强度、胁迫时期 内, 单位根质量分泌的可溶性糖随胁迫强度的增大 而增加; 超过此范围, 其分泌量逐渐降低。

(3) $\mathrm{BaP}$ 胁迫促进了根系对低分子量有机酸的 释放。修复潜力越强, 其高峰值出现的胁迫强度越 大、不同胁迫期内的释放量差异也越显著; 胁迫期 越长, 释放量越低。其中, 草酸、乙酸、乳酸和苹果 酸是主要组分, 其质量分数超过了有机酸总量的 $97.34 \%$, 在修复潜力较强的植物根系的分泌物中还 检测到反丁烯二酸。

(4) $\mathrm{BaP}$ 胁迫对根系分泌物中氨基酸的种类影 响不大, 但对分泌量影响较大。苏氨酸、丝氨酸、 甘氨酸、丙氨酸的释放量随着胁迫强度的增加而剧 增; 脯氨酸、羟脯氨酸和天冬氨酸则近乎以加和效 应甚至协同效应的形式参与植物对 $\mathrm{BaP}$ 胁迫的应激 反应, 参与响应的组分越多, 表现出的修复潜力越 强。

总体上, $\mathrm{BaP}$ 胁迫环境中, 5 种羊茅属植物根系 的几种低分子量有机物的释放特征与植物的修复潜 力有关, 修复潜力越强, 释放量越多且成分越复杂, 并表现出更强的环境适应性及生理可塑性。

基金项目 国家自然科学基金(31300439)和四川省 教育厅自然科学基金(16ZA0385)。

\section{参考文献}

Abhilash PC, Powell JR, Singh HB, Singh BK (2012). Plantmicrobe interactions: Novel applications for exploitation 
in multipurpose remediation technologies. Cell, 30, 416420.

Bertin C, Yang X (2003). The role of root exudates and allelochemicals in the rhizosphere. The Plant Soil, 256, 6783.

Chen SC, Liao CM (2006). Health risk assessment on human exposed to environmental polycyclic aromatic hydrocarbons pollution sources. Science of the Total Environment, 366, 112-123.

Chen SJ, Zhu XL, Feng XZ, Huang LQ, Mei YQ (2012). Effect of polycyclic aromatic hydrocarbons (PAHs) on plant. Bulletin of Biology, 45(2), 9-11. (in Chinese) [陈世军, 祝 贤凌, 冯秀珍, 黄烈琴, 梅运群 (2012). 多环芳烃对植 物的影响. 生物学通报, 45(2), 9-11.]

D’Orazio V, Ghanem A, Senesi N (2013). Phytoremediation of pyrene contaminated soils by different plant species. Clean-Soil, Air, Water, 41, 377-382.

Edwards J, Johnson C, Santos-Medellín C, Lurie E, Podishetty NK, Bhatnagar S, Jonathan AE, Sundaresan V (2015). Structure, variation, and assembly of the root-associated microbiomes of rice. Proceedings of the National Academy of Sciences of the United States of America, 112, 911-920.

Gao YZ, Ling WT, Wong MH (2006). Plant-accelerated dissipation of phenanthrene and pyrene from water in the presence of a nonionic-surfactant. Chemosphere, 63, 15601567.

Gao YZ, Ren LL, Ling WT, Gong SS, Sun BQ, Zhang Y (2010). Desorption of phenanthrene and pyrene in soils by root exudates. Bioresource Technology, 101, 1159-1165.

Howsam M, Jones KC, Ineson P (2000). PAHs associated with the leaves of three decidous tree species. I-Concentration and profile. Environmental Pollution, 108, 413-424.

Hunter PJ, Teakle GR, Bending GD (2014). Root traits and microbial community interactions in relation to phosphorus availability and acquisition, with particular reference to Brassica. Frontiers Plant Science, 2, 352-361.

Jennifer AK, Nina MG, Frank G (2015). Plant-soil interactions in metal contaminated soils. Soil Biology \& Biochemistry, 80, 232-236.

Joner EJ, Leyval C (2003). Rhizosphere gradients of polycyclic aromatic hydrocarbon (PAH) dissipation in two industrial soils and the impact of arbuscular mycorrhiza. Environmental Science \& Technology, 37, 2371-2375.

Kim YB, Park KY, Chung Y, Buchanan BB (2004). Phytoremediation of anthracene contaminated soils by different plant species. Journal of Plant Biology, 47, 174-178.

Kirk JL, Klironomos JN, Lee H, Trevors JT (2005). The effects of perennial ryegrass and alfalfa on microbial abundance and diversity in petroleum contaminated soil. Environmental Pollution, 133, 455-465.

Lakshmanan V, Selvaraj G, Bais HP (2014). Functional soil microbiome: Belowground solutions to an aboveground problem. Plant Physiology, 166, 689-700.

Li XH, Ma LL, Liu XF, Fu S, Cheng HX, Xu XB (2006). Polycyclic aromatic hydrocarbon in urban soil from Beijing, China. Journal of Environmental Sciences, 18, 944-950.

Line ES, Paul HK, Torben N, Christian K, Jørgen S (2003). Toxicity of eight polycyclic aromatic compounds to red clover (Trifolium pratense), ryegrass (Lolium perenne) and mustard (Sinapsis alba). Chemosphere, 53, 993-1003.

Liu H, Ye YB, Cui B, Zheng LM, Huang YH, Wang ZH (2008). Responses of Arabidopsis thaliana to oxidative stress induced by polycyclic aromatic hydrocarbon fluoranthene. Chinese Journal of Applied Ecology, 19, 413-418. (in Chinese with English abstract) [刘泓, 叶媛 蓓, 崔波, 郑荔敏, 黄炎和, 王宗华 (2008). 多环芳烃 荧葱诱导拟南芥氧化胁迫. 应用生态学报, 19 , 413-418.]

Liu J, Zhou ML, Zhang N, Chen GP, Zhao RR, Gao X, Shi FC (2015). Effects of polycyclic aromatic hydrocarbons (phenanthrene and pyrene) on the growth and physiological cheracteristics of Spartina atterniflora. Acta Scientialiu Universitatis Nankaiensis, 48(1), 14-20. (in Chinese with English abstract) [刘静, 周美利, 张楠, 陈 国平, 赵瑞瑞, 高金金, 石福臣 (2015). 多环芳烃菲和芘 对互花米草生长和生理特征的影响. 南开大学学报(自 然科学版), 48(1), 14-20.]

Liu Y, Zhou LL (2011). Comparative studies on absorption of benzo (a) pyrene $(\mathrm{BaP})$ in city atmosphere by leaves of 8 street plants. Urban Environment \& Urban Ecology, 24(4), 5-8. (in Chinese with English abstract) [刘玉, 周璐 璐 (2011). 8种植物叶片对城市大气苯并(a)芘(BaP)的吸 收比较. 城市环境与城市生态, 24(4), 5-8.]

Luo L, Zhang SZ, Shan XQ, Zhu YG (2006). Oxalate and root exudates enhance the desorption of p,p'-DDT from soils. Chemosphere, 63, 1273-1279.

Maliszewska-Kordybach B, Smreczak B (2000). Ecotoxicological activity of soils polluted with polycyclic aromatic hydrocarbons (PAHs)—Effect on plants. Environmental Technology, 21, 1099-1110.

Marschner P, Crowley D, Yang CH (2004). Development of specific rhizosphere bacterial communities in relation to plant species, nutrition and soil type. The Plant Soil, 261, 199- 208.

Mueller KE, Shann JR (2006). PAH dissipation in spiked soil: Impacts of bioavailability, microbial activity, and trees. Chemosphere, 64, 1006-1014.

Pan SW, Wei SQ, Yuan X, Cao SX (2008). The removal and remediation of phenanthrene and pyrene in soil by mixed cropping of alfalfa and rape. Agricultural Sciences in China, 7, 1355-1364.

Phillipsa LA, Greerb CW, Farrella RE, Germidaa JJ (2012). 
Plant root exudates impact the hydrocarbon degradation potential of a weathered-hydrocarbon contaminated soil. Applied Soil Ecology, 52, 56-64.

Ramos R, Garcia E (2007). Induction of mixed-function oxygenase system and antioxidant enzymes in the coral Montastraea faveolata on acute exposure to benzo (a) pyrene. Comparative Biochemistry and Physiology Part C: Toxicology \& Pharmacology, 144, 348-355.

Shahzad T, Chenu C, Genet P, Barot S, Perveen N, Mougin C, Fontaine S (2015). Contribution of exudates, arbuscular mycorrhizal fungi and litter depositions to the rhizosphere priming effect induced by grassland species. Soil Biology \& Biochemistry, 80, 146-155.

Shen H, Yan XL, Zheng SL, Wang XR (2002). Exudation and accumulation of citric acid in common bean in response to Al toxicity stress. Chinese Journal of Applied Ecology, 13, 307-310. (in Chinese with English abstract) [沈宏, 严小 龙, 郑少玲, 王秀荣 (2002). 铝毒胁迫诱导菜豆柠檬酸 的分泌与累积. 应用生态学报, 13, 307-310.]

Sumia K, Muhammad A, Samina I, Qaiser K (2013). Plantbacteria partnerships for the remediation of hydrocarbon contaminated soils. Chemosphere, 90, 1317-1332.

Sun TR, Cang L, Wang QY, Zhou DM, Cheng JM, Xu H (2010). Roles of abiotic losses, microbes, plant roots, and root exudateson phytoremediation of PAHs in a barren soil. Journal of Hazardous Materials, 176, 919-925.

Susarla S, Medina VF, McCutcheon SC (2002). Phytoremediation: An ecological solution to organic chemical contamination. Ecological Engineering, 18, 647-658.

Tao S, Cui YH, Xu FL, Li BG, Cao J, Liu WX, Schmitt G, Wang XJ, Shen WR, Qing BP, Sun R (2004). Polycyclic aromatic hydrocarbons (PAHs) in agricultural soil and vegetable from Tianjin. Science of the Total Environment, $320,11-24$.

Tejeda-Agredano MC, Gallego S, Vila J, Grifoll M, Ortega-Calvo JJ, Cantos M (2013). Influence of the sunflower rhizosphere on the biodegradation of PAHs in soil. Soil Biology \& Biochemistry, 57, 2065-2076.

Tian XX, Zhou GY, Peng PA (2008). Concentrations and influence factors of polycyclic aromatic hydrocarbons in leaves of dominant species in the Pearl River Delta, South China. Environmental Science, 29, 849-854. (in Chinese with English abstract) [田晓雪, 周国逸, 彭平安 (2008). 珠江三角洲地区主要树种叶片多环芳烃含量特征及影 响因素分析. 环境科学, 2008, 29, 849-854.]

Tu SX, Ma L, Thomas L (2004). Root exudates and arsenic accumulation in arsenic hyperaccumulating Pteris vittata and non-hyperaccumulating Nephrolepis exaltata. Plant and Soil, 258, 9-19.

Wang HC, Hu LL, Li M, Chen WF, Wang Y, Zhou JJ (2013). Growth effects and accumulations of polycyclic aromatic hydrocarbons (PAHs) in rape. Chinese Journal of Plant Ecology, 37, 1123-1131. (in Chinese with English abstract) [王海翠, 胡林林, 李敏, 陈为峰, 王䒯, 周佳佳 (2013). 多环芳烃(PAHs)对油菜生长的影响及其积累效 应. 植物生态学报, 37, 1123-1131.]

Wei SQ, Pan SW (2010). Phytoremediation for soils contaminated by phenanthrene and pyrene with multiple plant species. Journal of Soils and Sediments, 10, 886-894.

Wu LK, Lin XM, Lin WX (2014). Advances and perspective in research on plant-soil-microbe interactions mediated by root exudates. Chinese Journal of Plant Ecology, 38, 298310. (in Chinese with English abstract) [吴林坤，林向民， 林文雄 (2014). 根系分泌物介导下植物-土壤-微生物互 作关系研究进展与展望. 植物生态学报, 38, 298-310.]

Xie XM, Liao M, Yang J (2011). Effects of pyrene on low molecule weight organic compounds in the root exudates of ryegrass (Lolium perenne L). Acta Ecologica Sinica, 31, 7564-7570. (in Chinese with English abstract) [谢晓梅, 廖敏, 杨静 (2011). 萠对黑麦草根系几种低分子量有机 分泌物的影响. 生态学报, 31, 7564-7570.]

Xie XM, Liao M, Yang J, Chai JJ, Fang S, Wang RH (2012). Influence of root-exudates concentration on pyrene degradation and soil microbial characteristics in pyrene contaminated soil. Chemosphere, 88, 1190-1195.

Xu C, Lin XF, Xia BC (2010). Response of root exudates of maize seedlings (Zea mays L.) to pyrene contamination. Acta Ecologica Sinica, 30, 3280-3288. (in Chinese with English abstract) [许超, 林小方, 夏北成 (2010). 玉米 幼苗根系分泌物对芘污染的响应. 生态学报, 30, 32803288.]

Yi H, Crowley DE (2007). Biostimulation of PAH degradation with plants containing high concentrations of linoleic acid. Environmental Science \& Technology, 41, 4382-4388.

Zhu YH, Zhang SZ, Huang HL, Wen B (2009). Effects of maize root exudates and organic acids on the desorption of phenanthrene from soils. Journal of Environmental Sciences, 21, 920-926.

责任编委: 段昌群 责任编辑: 李 敏 


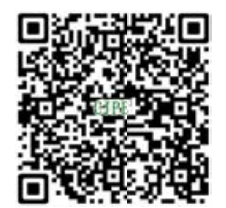

植物生态学报官网

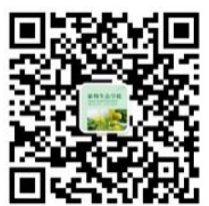

微信订阅号

期刊及学科

相关信息发布

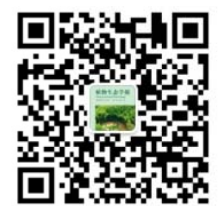

微信服务号

稿件状态查询

全文检索浏览 